
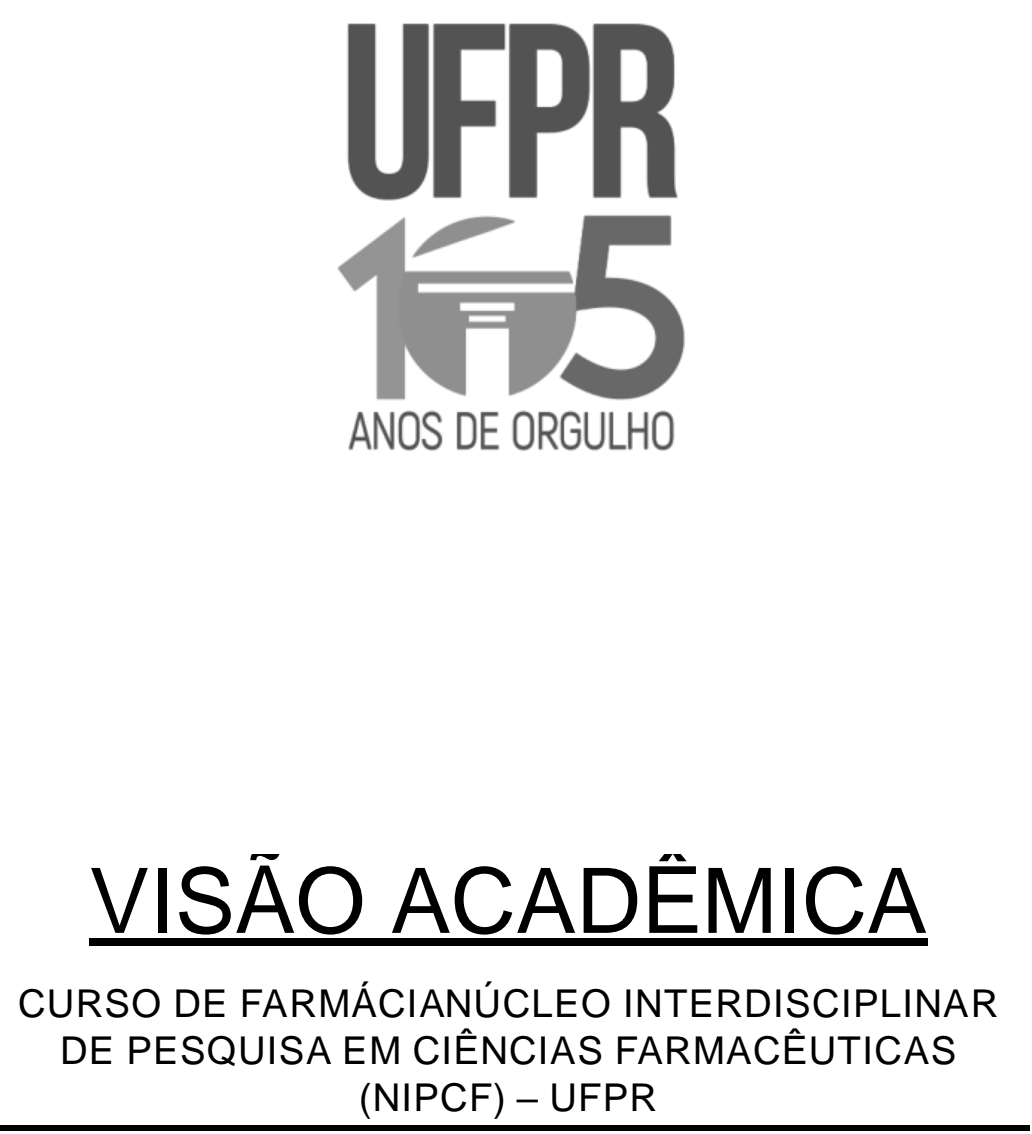

O Sistema Eletrônico de Revistas (SER) é um software livre e permite a submissão de artigos e acesso às revostas de qualquer parte do mundo. Pode ser acessado por autores, consultores, editores, usuários, interessados em acessar e obter cópias de artigos publicados nas revistas. O sistema avisa automaticamente, por e-mail, do lançamento de um novo número da revista aos cadastrados. 


\section{VISÃO ACADÊMICA}

Reitor

Ricardo Marcelo Fonseca

Direção do Setor de Ciências da Saúde
Claudete Reggiani

Vice-Direção do Setor de Ciências da Saúde Nelson Luis Barbosa Rebellato

\section{Corrdenador(a) do Curso de Farmácia}

Nilce Nazareno da Fonte

Núcleo Interdisciplinar de Pesquisa em Ciências Farmacêuticas

Coord. Maria Madalena Gabriel

Editor Responsável

Obdulio Gomes Miguel (UFPR)

\section{Coordenador Editorial}

Marilis Dallarmi Miguel (UFPR)

\section{Comissão Editorial}

Maria Madalena Gabriel (UFPR)

Sandra Maria Warumbi Zanin (UFPR)

Marilu Lopes (UFPR)

Roberto pontarolo (UFPR)

Eliane Carneiro Gomes (UFPR)

Vitor Alberto Kerber (UFPR)

Marlene Maria Fregonezi Nery (UEL)

Sandra Mara Woranovicz Barreira (UFPR)

Rogério Luiz Koop (UFPR)

\section{Comissão de Publicação}

Josiane de Fatima Gaspari Dias (UFPR) Patrícia

T. P. S. Pentado (UFPR)

Eliane Rose Serpe (UFPR)

Jorge Guido Chociai (UFPR)

Marilene da Cruz Magalhães Bufon(UFPR)

Cristiane Bezerra da Silva (UFPR)

\section{Ficha Catalográfica}

Visão Acadêmica / Nucleo Intersdiciplinar de Pesquisa em Ciências Farmacêuticas da UFPR - Vol. 19, n. 4 - Curitiba, 2018 - Trimestral.

ISSN 1518-5192

1. Farmácia

2. Universidade Federal do Paraná. Núcleo Intersdiciplinar de Pesquisa em Ciências Farmacêuticas.

CDD615

INDEXAÇÃO: LATINDEX - Directório de Publicaciones Científicas Seriadas de América Latina. El Caribe, Espanã Y Portugal;

\author{
Comissão de Divulgação \\ Vinicius Bednarczuk de Oliveira (UFPR) \\ Mariana Saragioto Krause (UFPR) \\ Cristiane da Silva Paula (UFPR) \\ Francis José Zortéa Merino (UFPR) \\ Beatriz Cristina Konopatzki Hirota(UFPR) \\ Conselho Consultivo \\ Adair Roberto Santos (UFSC) \\ Amélia Therezinha Henriques (UFRGS) \\ João Batista Calixto (UFSC) \\ Luiz Doni Filho (UFPR) \\ Ricardo Andrade Rebelo (FURB) \\ Carlos Cezar Stadler (UEPG) \\ Rosendo Augusto Yunes (UFSC) \\ Valquíria Linck Bassani (UFRGS) \\ Raquel Rejane Bonato Negrelle (NIMAD - UFPR) \\ Grace M.C. Wille (UFPR)

\section{Conselho Externo} \\ Franco Delle Monache \\ Instituto di Chimica, Universitá Cattolica, Roma - Itália \\ Sixto Hugo Rabery Cáceres \\ Facultad de Ingeniería Agronómica-UNE, Assuncion -

$$
\text { Paraguai }
$$ \\ Fernando Fernández - Llimós \\ Universidad de Granada - Espanha
}

VISÃO ACADÊMICA é a revista do Núcleo Interdisciplinar de Pesquisa em Ciências Farmacêuticas (NIPCF) - do curso de Farmácia - UFPR, editada semestralmente com duas seções: Artigos Científicos, Artigos de Revisão e Resumo de DissertaçõeseTeses.

É permitida a reprodução com menção da fonte de artigos e fotos, sem reserva de direitos autorais.

Esta revista poderá ser obtida (solicitação ao Coordenador Editorial por doação ou permuta), junto à disciplina de farmacotécnica, Curso de Farmácia - UFPR.

Correspondências e artigos para publicação deverão ser encaminhados à: Universidade Federal do Paraná

Setor de Ciências da Saúde - Curso de Farmácia Departamento de Farmácia A/C Profa. Marilis Dallami Miguel

Rua Pref. Lothário Meissner, 632, Jardim Botânico, CEP 80210-170 - Curitiba - PR

Fone: (41) 3360-4070 / Fax: (41) 3360-4101 e-mail: visaoacd@ufpr.br

Bibliotecária

Clarice Siqueira Gusso

Secretário Científico

Paulo Sérgio Diniz

e-mail: psdiniz@ufpr.br 


\section{VISÃO ACADÊMICA}

CURSO DE FARMÁCIA NÚCLEO INTERDISCIPLINAR DE PESQUISA EM CIÊNCIAS FARMACÊUTICAS (NIPCF) - UFPR

\section{Sumário}

COMPOSIÇÃO CENTESIMAL E NUTRICIONAL DA MACADÂMIA (MACADAMIA INTEGRIFOLIA MAIDEN \& BETCH) E ANÁLISE DO SEU PERFIL LIPÍDICO RELACIONADO À TERAPÊUTICA MACADAMIA'S (MACADAMIA INTEGRIFOLIA MAIDEN \& BETCH) CENTESIMAL AND NUTRITIONAL COMPOSITION AND PROFILE ANALYSIS RELATED TO TERAPHEUTICS FEDALTO, M. B.; STOFELLA, N. C. F.; GOMES, D.L.; MAAS, N. C.; MUNOZ, M. M.; SILVA, M. S.; FLORENTINO, I.; BALBI, M. E.

CONDUTA DIETOTERÁPICA PARA PACIENTE COM GASTRITE CRÔNICA E OSTEOPOROSE: RELATO DE CASO

CONDUCT DIET THERAPEUTIC FOR PATIENT WITH CHRONIC GASTRITIS AND OSTEOPOROSIS: CASE REPORT

JULIANE ZENI; PATRICIA CHICONATTO; VANIA SCHMITT; CARYNA EURICH MAZUR. 19

USO DA FARINHA DA CASCA DO MARACUJÁ AMARELO (PASSIFLORA EDULIS F. FLAVICARPA DEG. - FAMÍLIA PASSIFLORACEAE) NA FORMULAÇÃO DE MACARRÃO CASEIRO

USE OF PASSIONFRUIT PEEL FLOUR (PASSIFLORA EDULIS F. FLAVICARPA DEG. - FAMILY PASSIFLORACEAE) IN HOMEMADE PASTA RECIPE.

B. F. COSTA; F. R. DE LIZ; J. G. S. FERREIRA; R. C. SANTOS; T. H. GONÇALVES; M. E. BALBI 32

ÍNDICES DE DESENVOLVIMENTO EM JUVENIS BETTA SPLENDENS ALIMENTADOS COM ADITIVOS ENZIMÁTICOS

DEVELOPMENTAL INDEXES IN JUVENILE BETTA SPLENDENS FOODS WITH ENZYMATIC ADDITIVES

VERUSKA DILYANNE SILVA GOMES; ALDA LÚCIA DE LIMA AMÂNCIO; JOSÉ JORDÃO FLLHO; CACIO RIBEIRO CAVALCANTI; JOÃO MARCOS MONTEIRO BATISTA; JOSÉ HUMBERTO VILAR DA SILVA.

MARKETING FARMACÊUTICO: RELAÇÃO DAS PUBLICIDADES TELEVISIVAS COM A AUTOMEDICAÇÃO

PHARMACEUTICAL MARKETING: RELATIONSHIP OF TELEVISION ADVERTISING WITH AUTOMEDICATION

LIANA MARIA MAZUTTI CARSONI; DEVANIR AVIGO JUNIOR 55

BENEFÍCIOS FUNCIONAIS DO CACAU (THEOBROMA CACAO) E SEUS DERIVADOS FUNCTIONAL BENEFIT OF COCOA (THEOBROMA CACAO) AND THEIR DERIVATIVES HELOISA DE OLIVEIRA RIBAS; DANYELLEN STAUT GONÇALVES; CARYNA EURICH MAZUR

FUNDAMENTOS DA NUTRIÇÃO NA PRÁTICA DE PILATESß: UMA REVISÃO DE LITERATURA NUTRITION FUDAMENTS IN PILATES $\AA$ PRACTICE: A LITERATURE REVIEW NEIDI APARECIDA LISBOA DA LUZ; CARYNA EURICH MAZUR; ALINE JABUR CASTLLHO 